\title{
Therapie mit elektromechanisch- assistierten Geräten: Tipps und Tricks für die praktische Umsetzung
}

\author{
Die roboterassistierte Gangtherapie sowie die roboterassistierte Armtherapie haben \\ sich zu einem festen Bestandteil der neurologischen Rehabilitation etabliert. Sie sind \\ für die Therapeuten entlastende und für Patienten sichere Therapieformen. Darüber \\ hinaus handelt es sich um effektive Methoden in der Physiotherapie, um das Gehen \\ nach einem Schlaganfall wiederzuerlernen und die Armfunktionen zu verbessern. Das \\ Erfolgsrezept dafür liegt vermutlich in der hohen Wiederholungszahl und der intensiven \\ und aktiven physischen Beanspruchung des Patienten. Simone Thomas und Jan Mehrholz
}

\section{Gangtherapie mit Roboter}

Beim Thema aktive Beanspruchung gibt es einen deutlichen Unterschied zwischen den beiden gängigen Systemen zur roboterassistierten Gangtherapie (RAGT), dem Endeffektor und dem Exoskelett. Während das Exoskelett z.B. die Kniestreckung innerhalb der Standbeinphase vollständig übernimmt, muss der Patient bei einem Endeffektorsystem die Bewegung aktiv selbst durchführen. Vor allem ein Training mit Endeffektorsystemen erscheint, laut einem aktuellen Cochrane-Review von 2017, im Vergleich zu Exoskeletten, zumindest in Bezug auf Gehgeschwindigkeit und Gangausdauer, effektiver.

Eine höhere Eigenaktivität des Patienten innerhalb einer RAGT kann daher durchaus sinnvoll sein. Näher erläutert hat der Roboter die Aufgabe, das Gehen zu simulieren, während der Patient versucht, diesen Prozess aktiv zu übernehmen. Jedoch ist es teilweise eine Herausforderung, die Konzentration des Patienten während der RAGT stets auf das aktive Gehen zu lenken. Hier können Vorstellungsbilder wie Wandern oder auch Musik während des Trainings die Freude am Üben erhalten, was sich förderlich auf das motorische Lernen auswirkt [20]. Weiterhin unterstützt ein externer Fokus innerhalb der Aufgabenstellung die Bewegungsoptimierung und die Konzentration [20]. Denn je intensiver die Gehbewegung aktiv wiederholt wird, desto eher ist es wahrscheinlich, dass der Patient gehen lernt.

Wer profitiert von der roboterassistierten Gangtherapie?

Schlaganfall Das Training mittels Roboter ist vor allem für Patienten nach einem Schlag- anfall geeignet. Bei keinem anderen neurologischen Krankheitsbild wurden die Effekte der RAGT so deutlich und häufig durch klinische Studien untersucht und belegt [11]. Effektiv ist das Training jedoch vor allem, wenn der Patient nach einem Schlaganfall noch nicht selbstständig gehen kann (Functional Ambulation Categories 0-2) (Abb. 1) und wenn innerhalb der ersten drei Monate nach dem Akutereignis geübt wird [11].

Multiple Sklerose Weiterhin konnte eine kleine Pilotstudie, in der Patienten mit Multipler Sklerose (Expanded Disability Status Scale 6-7,5) mittels RAGT trainierten, positive Effekte auf die Gehgeschwindigkeit, die Ausdauer und die Kniestreckung ermitteln [1].

Parkinson-Erkrankung Zur Verbesserung der Gehgeschwindigkeit und Balance kann auch bei Patienten mit Morbus Parkinson (Hoehn-und-Yahr-Skala 1-4) der Gangroboter mit hoher Gehgeschwindigkeit zum Einsatz kommen [16]. Jedoch ist die schnelle Laufbandtherapie für die Optimierung des Ganges bei Morbus Parkinson vorzuziehen [8]. Der Fokus in der Gangtherapie von Patienten mit Parkinson liegt nicht auf dem Lernen einer Bewegung durch eine hohe Wiederholungszahl, sondern eher auf dem Üben mit hoher Geschwindigkeit und vergrößerter Bewegungsamplitude. Die Laufbandtherapie bietet für Patienten mit Morbus Parkinson ein Training mit höherer Gehgeschwindigkeit und mit einer vergrößerten Schrittlänge. Zwei wesentliche Therapiefaktoren, die innerhalb der RAGT stark begrenzt sind.
Infantile Zerebralparese Weiterhin können auch Kinder mit Zerebralparese (Gross Motor Function Level 1-4) von der RAGT profitieren $[3,12,18]$. In den dazu durchgeführten kleineren Studien konnten deutliche Effekte auf Gehgeschwindigkeit und Ausdauer ermittelt werden. Interessanterweise scheint hier ein erschwerendes Gehtraining (Gehen gegen Widerstände mittels Roboter) effektiver zu sein als ein assistierendes RAGT, um eine Verbesserung des Gehens zu erreichen [19].

Querschnittläsion Im Gegensatz dazu profitieren Patienten mit Querschnittläsion nicht unbedingt von der RAGT. Es ist bislang noch relativ unklar, ob eine rein konventionelle Gangtherapie nicht mindestens genauso bedeutende Effekte auf das Gehen erzielt wie RAGT [10].

Es kann also geschlussfolgert werden, dass eine RAGT v. a. bei Patienten, die akut oder subakut vom Schlaganfall betroffen sind und nicht selbstständig gehen können, angewandt werden sollte. Kleinere Studien zeigen ebenfalls Effekte bei Patienten mit Multipler Sklerose und Kindern mit Infantiler Zerebralparese, die leichte bis deutliche Geheinschränkungen aufweisen.

Kontraindikationen Neben diesen Indikationen gibt es auch eine Reihe an Nebenerkrankungen und Symptomen, bei denen von einer RAGT abgeraten wird. Diese Ausschlusskriterien sind in Tab. 1 aufgeführt.

\section{Robotergestütztes Ausdauertraining} Gerade bei Patienten mit neurologischem Krankheitsbild ist die allgemeine Belastungsfähigkeit deutlich reduziert. Vor allem immobile Patienten kommen hinsichtlich der körperlichen Belastung sehr zeitig an ihre Erschöpfungsgrenze, was den Alltag oder auch das tägliche Üben erschwert. Das Training der Grundlagenausdauer durch kontinuierliche aerobe Belastung sollte somit ein bedeutender Bestandteil innerhalb der Therapie von neurologischen Patienten sein. Häufig ist es aber schwer, mit deutlich immobilen Patienten ein aerobes Ausdauertraining durchzuführen. Die RAGT eignet sich in der Praxis neben dem motorischen Gehtraining auch für ein kardiales Ausdauertraining. Voraussetzung hierfür ist die Herz-Kreislauf-Stabilität im Stand. 


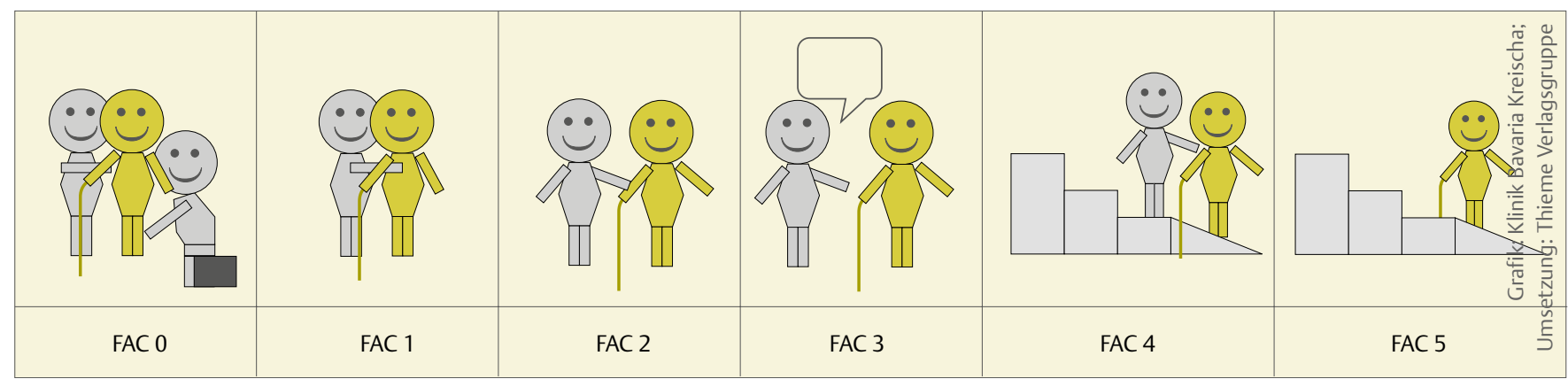

Abb. 1 Functional Ambulation Categories

\begin{tabular}{|c|c|c|}
\hline \multicolumn{3}{|c|}{ UEX = untere Extremität; NYAH= New York Heart Association; RR= Blutdruck } \\
\hline Bereich & Kriterien & Quelle \\
\hline \multirow[t]{4}{*}{ orthopädisch } & Kontrakturen, Entzündungen, Frakturen UEX, Wirbelsäule & {$[2,5,12,15,18]$} \\
\hline & $\begin{array}{l}>2 \mathrm{~cm} \text { Beinlängenunterschied (nicht durch Orthesen } \\
\text { ausgleichbar) }\end{array}$ & [3] \\
\hline & schwere Osteoporose & {$[12]$} \\
\hline & schwere Skoliose (Cobb-Winkel $\left.>20^{\circ}\right)$ & {$[12]$} \\
\hline \multirow[t]{3}{*}{ vaskulär } & Phlebothrombose Becken, UEX & {$[12]$} \\
\hline & Entzündungen, offene Wunden an Rumpf, UEX & {$[3,12,13]$} \\
\hline & Amputationen UEX ohne Prothese & {$[6]$} \\
\hline \multirow[t]{2}{*}{ kardiologisch } & Herzinsuffizienz (NYHA III-IV) & {$[5,12,15]$} \\
\hline & RR $>200 \mathrm{mmHg}$ systolisch, $110 \mathrm{mmHg}$ diastolisch & {$[6]$} \\
\hline neurologisch & allgemeiner Schwindel und Übelkeit & {$[16,17]$} \\
\hline weitere & nicht kontrollierbarer Diabetes mellitus & {$[6]$} \\
\hline
\end{tabular}

Um die minimale und maximale Grenze der Herzfrequenz für ein kardiales Training im Gangroboter zu bestimmen, kann die Karvonen-Formel genutzt werden [7].

Die durchschnittliche Dauer für ein kontinuierliches aerobes Ausdauertraining beträgt im Idealfall ca. 20 Minuten (mindestens 15 und maximal 30 Minuten) [4]. Außerdem empfiehlt es sich, dem Patienten eine Pulsuhr anzulegen, da sonst das Training durch manuelle Herzfrequenzmessungen häufig unterbrochen wird.

Um während des Trainings innerhalb der bestimmten Herzfrequenzgrenzen zu bleiben, können vier verschiedene Trainingsfaktoren angepasst werden:

- Gehgeschwindigkeit

- Körpergewichtsbelastung und -entlastung mittels Gurtsystem

- Eigenaktivität des Patienten

- Trainingsdauer

Je nach Leistungsgrenze des Patienten sollte erfahrungsgemäß mindestens einer der vier Trainingsfaktoren pro Therapieeinheit gesteigert werden.

\section{Therapieintensität}

Dauer und Inhalt der RAGT können je nach motorischem und kardialem Zustand des Patienten variieren. Aus Studien und Erfahrungswerten heraus kann argumentiert werden, dass der Patient drei bis fünf Mal pro Woche für je durchschnittlich 20 Minuten mit dem Roboter das Gehen trainieren sollte. Dies stellt eine hohe Wiederholungszahl sicher. Bei einem Schritt pro Sekunde geht der Patient innerhalb der 20 Minuten Therapie teilweise bis zu 1200 Schritte. Darüber hinaus sollte laut Studienlage zusätzlich ein konventionelles Steh- und Gehtraining von 20 bis 30 Minuten pro Therapieeinheit durchgeführt werden, da die RAGT nicht die alleinige Therapie sein sollte [5, 15].

\section{Gurtsystem richtig anlegen}

Die RAGT beginnt mit der Gurtanlage. Je nach Mobilitätsgrad und Gurtsystem kann dies im Liegen bei Sitzinstabilität, im Sitz bei nicht stehfähigen Patienten oder im Stand erfolgen. Nach der Gurtanlage ist im Stand zu prüfen, ob die Hüftbewegung in Extension blockiert oder die Oberkörperaufrichtung eingeschränkt ist (Abb. 2). Diese beiden „Gurtungsfehler“ passieren erfahrungsgemäß am häufigsten bei der Anlage im Sitz. Weiterhin sollte die Last des Oberkörpers mittels Gurtsystem nicht über die Oberarme abgefangen werden. Therapeuten sollten daher darauf achten, dass die ventralen Schulterriemen parallel zum Arm verlaufen und der Abstand zwischen Gurt und Schulter nach kranial maximal vier Finger breit ist (Abb. 3).

\section{Möglicher Therapieablauf}

Mit der RAGT kann bei Patienten nach Schlaganfall bereits innerhalb der ersten zwei Wochen nach dem Akutereignis begonnen werden [14]. Je frühzeitiger die Therapie innerhalb der Rehabilitation beginnt, desto besser. Die ersten Minuten der RAGT können zur Aufwärmung mit geringer Gehgeschwindigkeit genutzt werden. Hierbei sollten die Gehbewegung und die Gurtanlage erneut überprüft werden. Die Körpergewichtsentlastung und die Schrittlänge werden angepasst, während sich der Patient langsam an die Schrittfolge gewöhnt.

Bei Endeffektorsystemen sollte eine Gehgeschwindigkeit gewählt werden, bei welcher der Patient noch aktiv bzw. aktiv-assistiv die Stand- und Schwungbeinphase durchführen kann.

\section{Für einen guten Trainingseffekt sollte stets die Pulsfrequenz kontrolliert und mit den berechneten Pulsgrenzen verglichen werden.}

Nach der Trainingsphase erfolgt eine kurze Abwärmphase, bei der die Gehgeschwindigkeit auf das Anfangsniveau angepasst wird.

\section{Hilfestellungen in der Therapie}

Bei Endeffektorsystemen kann der Therapeut das Kniegelenk in Beugung und Stre- 
ckung unterstützen und stabilisieren, falls die Parese des Beines zu hoch ist.

Beide Hände können dabei mit dem Handteller proximal der Kniescheibe platziert werden. Die Finger umgreifen jeweils medial und lateral den Oberschenkel. Für die Kniestreckung wird Druck auf den Oberschenkel nach dorsal gegeben, für die Flexion greifen die Fingerspitzen in die Kniekehle und ziehen das Kniegelenk nach ventral. Bei dieser Grifffassung kann gleichzeitig das Kniegelenk stabilisiert werden (Abb. 4).

Einige Endeffektorgeräte haben auch Knieführungssysteme, welche z.B. aus elastischen Gurtungen bestehen. Je mehr der Patient aktiv übernehmen kann, desto weniger Hilfestellung sollte gegeben werden. Weiterhin können Bandagen oder spezielle Wickeltechniken Gelenkabweichungen oder Instabilitäten v. a. an Sprung- und Kniegelenk während der RAGT korrigieren. Bei Exoskeletten übernimmt der Roboter die Gelenkführung vollständig.

Aufmerksamkeit während des Gehtrainings Um die Aufmerksamkeit des Patienten auf das Gehen zu lenken und die Bewegung optimal zu lernen, kann ein externer Fokus innerhalb der Aufgabenstellung verwendet werden [20]. Für die Kniestreckung kann die Aufgabenstellung „Schieben Sie die Fußplatte nach vorn“, für die Beugung „Die Fußplatten nach hinten ziehen! “ lauten.

Eine andere Idee ist es, einen Punkt auf die Kniekehle zu kleben oder bei instabilem Kniegelenk das Knie mit einer Bandage zu umwickeln und in der Kniekehle einen kleinen Ball mit einzuwickeln. Der Patient soll nun die Gegenstände nach hinten wegschieben (Kniestreckung) und diese danach wieder nach vorn holen (Kniebeugung).

Auch verstärkte Vorstellungsbilder wie Skilanglauf oder einen Berg hochwandern helfen dabei, die Bewegung bestmöglich auszuführen. Modern und sinnvoll scheint auch die virtuell-visuelle Rückmeldung. In unserem Beispiel in Abb. 5 erkennt der Patient an einem Monitor, wie stark er das jeweilige Bein in der Standbeinphase belastet.

\section{Pausen im Gangroboter}

Pausen sollten so wenig wie möglich Teil der Therapie sein. Ist es dem Patienten jedoch nicht möglich, 20 Minuten am Stück zu trainieren, können ein bis zwei kurze Unterbrechungen während des Trainings stattfinden. Innerhalb der Pause ist es sinnvoll,
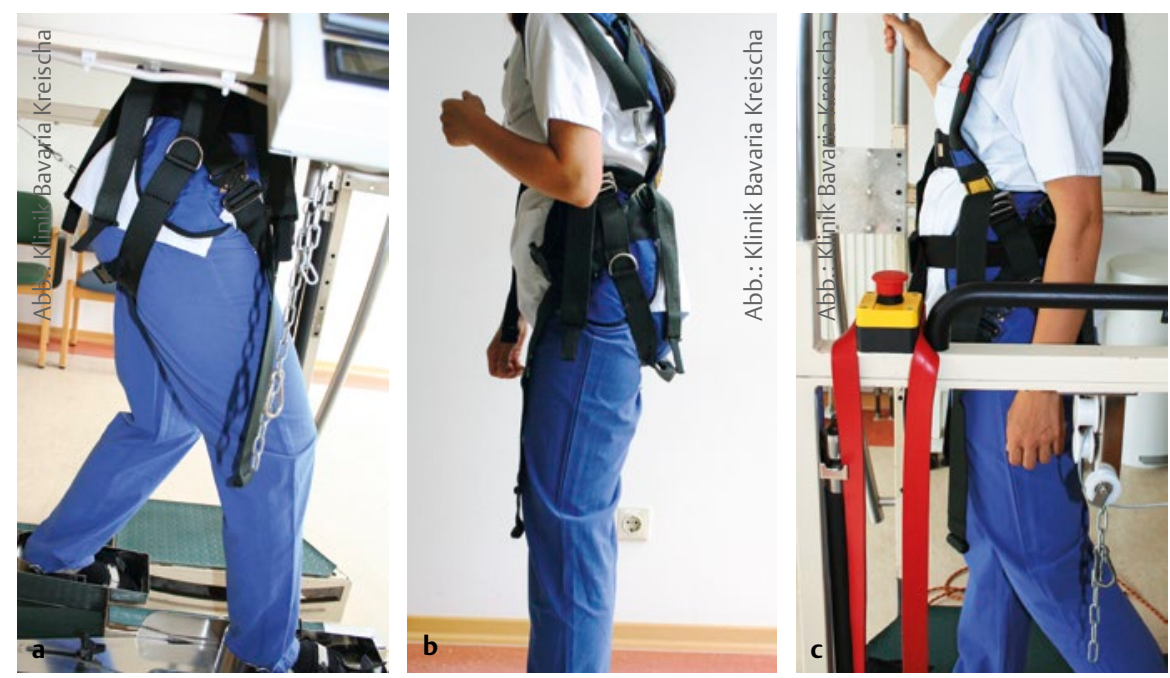

Abb. 2 Roboterassistierte Gangtherapie: Gurtanlage. a Hüftgelenk in der Standbeinphase wird in Extension eingeschränkt. b Oberkörper kann nicht aufgerichtet werden. c So sitzt der Gurt richtig.
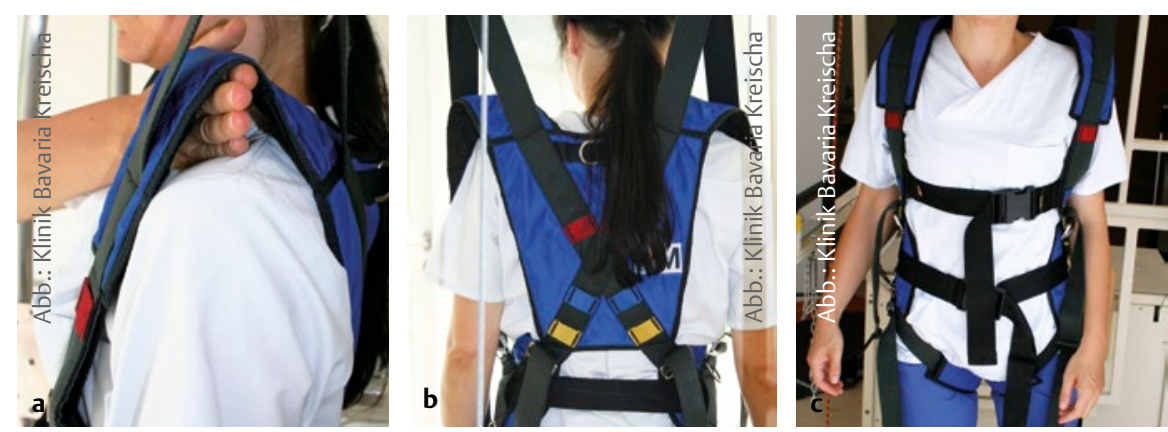

Abb. 3 Roboterassistierte Gangtherapie: Anlage der Schulterriemen. a Vier Finger passen unter den Gurt. b Schultergurt steht zu weit von der Schulter ab. c Oberarm fängt Gewicht des Oberkörpers ab.

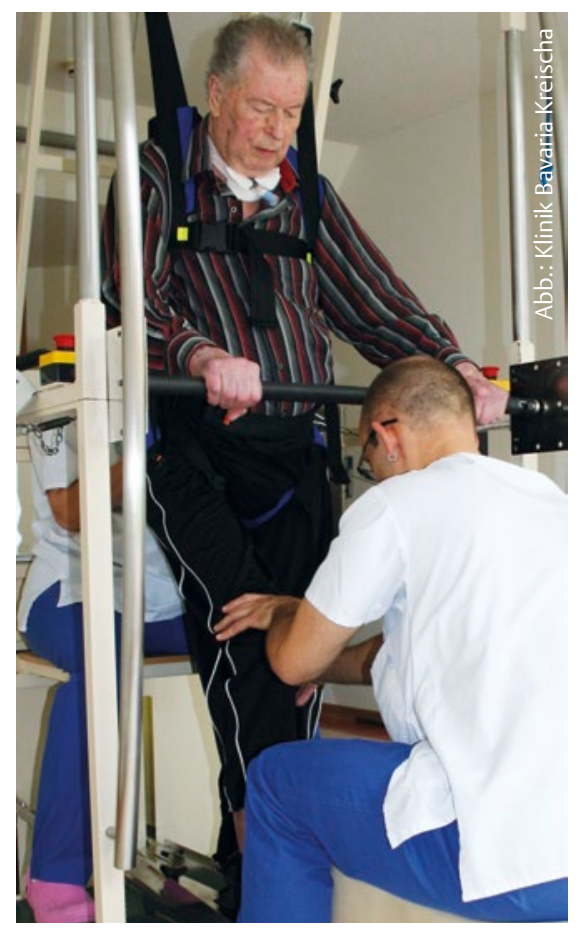

Abb. 4 Hilfestellung am Knie

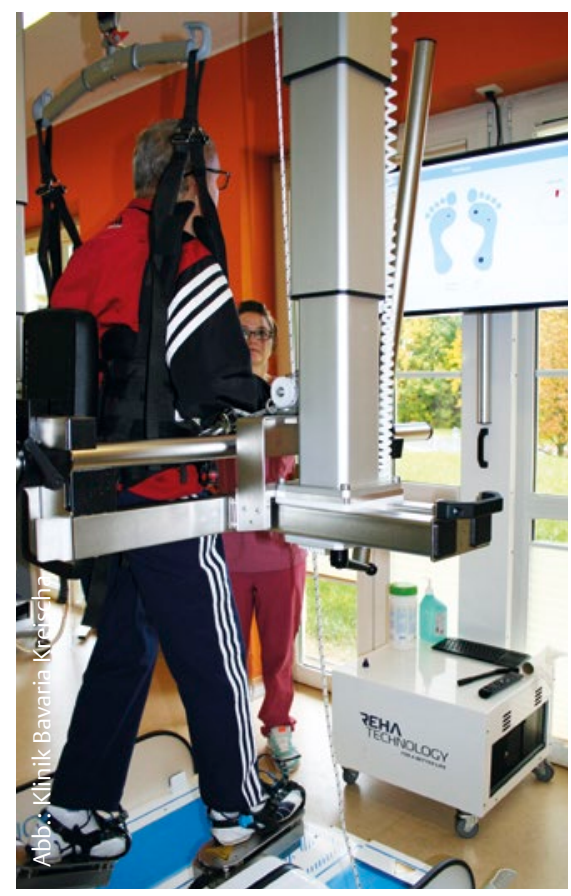

Abb. 5 Externer Fokus während des Gehens. Der Patient achtet auf den Bildschirm. 
das Gehen auf einem Video z. B. mittels Tablet oder bei anderen Personen zu beobachten. Auch mentale Strategien können zur Förderung des Lernens beitragen. Zum Beispiel soll der Patient die Augen schließen und sich vorstellen, wie er aus der Wohnungstür tritt, rechts die Straße vor seiner Wohnung entlangläuft, die Straßenseite wechselt usw. Sitzpausen sollten nur bei starker Blutdruckabweichung, vegetativen Zeichen einer Überbelastung oder wenn es der Patient ausdrücklich wünscht durchgeführt werden. Hier können passive Dehnungen der Waden- und Fußmuskulatur oder Lockerungsübungen für die Oberschenkelmuskulatur erfolgen.

\section{Armhaltung während der RAGT}

Patienten mit schlaffer Armlähmung oder Armschmerz haben ein großes Verletzungsrisiko der Extremität während der RAGT, da sich bei hoher Gehgeschwindigkeit der Arm unkontrolliert bewegt und sich der vorhandene Schmerz steigern kann. Zu empfehlen ist daher das Tragen einer Dreipunkt-Schulterschlinge während der Therapie.

Kann der Patient mit der paretischen Hand eine Faust andeuten und den Arm anheben, können Fixierungsmanschetten den Faustschluss unterstützen, wodurch der Patient sich an einer frontalen oder seitlichen Haltestange anhalten kann. Der Arm ist hier ebenfalls vor Verletzungen geschützt.

Damit der Patient keine Schmerzen im Arm entwickelt, müssen Höhe und Entfernung der Haltestange angepasst werden. Ist der Arm in Adduktions-Flexions-Haltung muskulär oder gelenkig fixiert, ist kein zusätzlicher Schutz des Armes notwendig. Der Arm sollte einfach in dieser Stellung verbleiben.

\section{Arm und Training -}

\section{Armtherapie mit Roboter}

Die roboterassistierte Armtherapie (RAAT) beinhaltet die gleichen Therapieansätze wie die RAGT: eine hohe Wiederholungszahl bei aufgabenspezifischem aktivem Üben. Vor allem das aktive Üben bei schwer ausgeprägten Paresen stellt häufig eine Herausforderung dar.

Die Motivation der Patienten ist aufgrund des ausbleibenden schnellen Therapieerfolges oft gering, und die Therapieideen sind begrenzt. Hier bietet die Technologie Hilfe. Es gibt diverse Geräte (z. B. ARMin ${ }^{\circledR}$, InMotion ARM $^{\circledR}$, WRIST ${ }^{\circledR}$ und HAND ${ }^{\circledR}$, ARMEO-

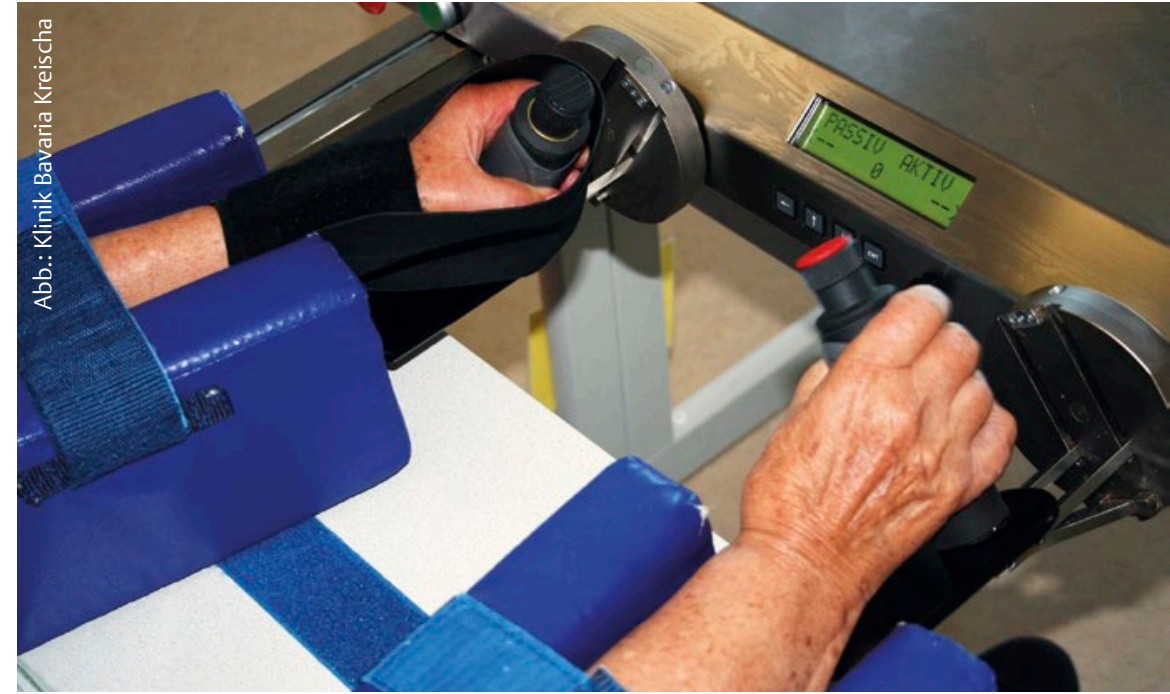

Abb. 6 Der Monitor zeigt die Anzahl der Bewegungen an.

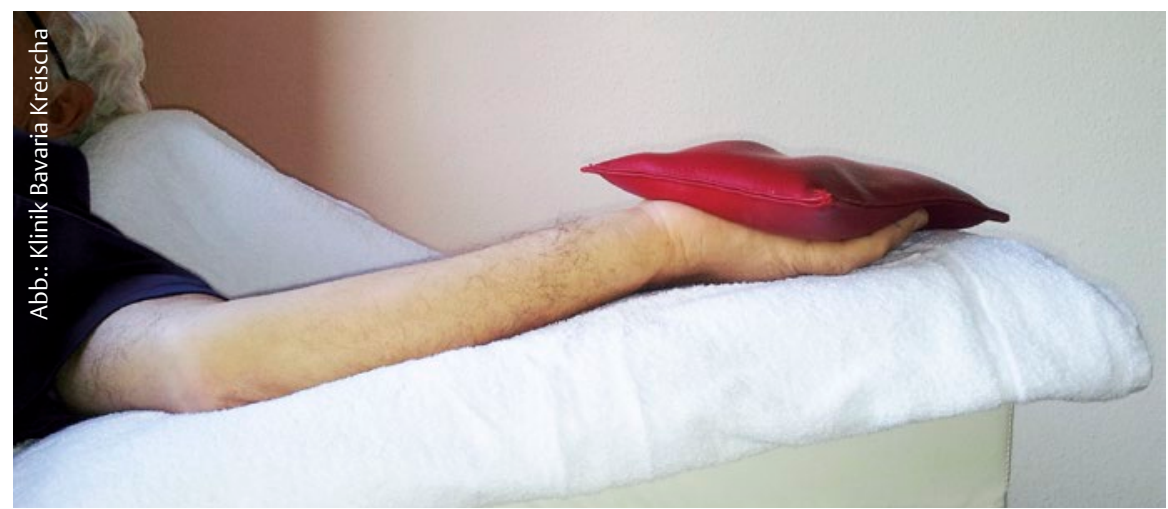

Abb. 7 Dehnlagerung Hand-und Fingerflexoren.

spring $^{\circledR}$, BI-MANU-Track ${ }^{\circledR}$ ), die den Patienten bilateral und unilateral, distal oder proximal betont, selektiv oder komplex sowie passiv oder assistiv unterstützten.

Auch hier profitieren v.a. Patienten mit akutem Schlaganfall (<drei Monate) hinsichtlich Alltagsleistungen, Armfunktion und Armkraft [9]. Ein zeitiger Beginn innerhalb der Rehabilitation ist daher wesentlich.

Vor allem Geräte, welche die Finger, Hände und Unterarme unterstützen, haben einen bedeutenden Therapieeffekt auf den Alltag der Patienten [9]. Beim RAAT gibt es insgesamt sehr gute Evidenz, allerdings sind die durch die Studien zu erwartenden Effekte geringer und nicht so sehr ausgeprägt wie beim RAGT.

Virtuelle Realität und Aufmerksamkeit bei der Armtherapie

Häufig wird die RAAT auch mit virtueller Realität (VR) verknüpft. Dies hat den Vorteil, dass die Patienten beim Üben motivierter sind, weil die Aufmerksamkeit länger auf jekt liegt. Aber auch ohne VR kann ein externer Fokus während des Übens in die Aufgabenstellung integriert werden. Auch die Motivation kann mittels der Aufgabenstellung und äußeren Feedbackmechanismen aufrechterhalten werden.

Hier ein Beispiel: Am BI-MANU-Track soll der Patient z.B. die Griffe so weit wie möglich nach innen und außen drehen. Die Konzentration liegt nun auf dem Gerät und nicht auf der Hand (externer Fokus). Zusätzlich kann der Patient ein Klacken hören, wenn die Bewegung ausreichend weit erfolgte (objektives externes Feedback). Der Patient soll nun in fünf Minuten so oft wie möglich die Griffe nach innen und außen drehen. Der Roboter zählt die erfolgreichen Bewegungen und belohnt den Patienten pro Bewegung mit einem erfolgreichen „Klick“. Anschließend hat er eine Minute Pause. Danach soll er die Bewegungsanzahl innerhalb der nächsten fünf Minuten halten oder erhöhen (Abb. 6). der Armaktivität oder einem externen Ob- 


\section{Ausschlusskriterien}

Die RAAT ist besonders bei von Schlaganfall betroffenen Patienten mit moderater bis schwerer Funktionsbeeinträchtigung der oberen Extremität indiziert. Patienten mit leichten Paresen haben häufig ein Schnelligkeits- und Koordinationsproblem der oberen Extremität, für das die RAAT nur selten ausreichend ausgelegt ist.

Eine Voraussetzung für die RAAT ist die Schmerzfreiheit bei der zu übenden Bewegung. Sobald Schmerzen eintreten, müssen die Geräteeinstellung oder die Gelenkstellungen von Schulter, Ellenbogen und Handgelenk überprüft werden.

Schwere Kontrakturen, die auch durch Dehnlagerungen vor der Therapie nicht den notwendigen Bewegungsradius für das $\mathrm{Ge}$ rät freigeben, sind ein Ausschlusskriterium für die RAAT.

Im Gegensatz zur Spastik: Erfahrungsgemäß kann hier das aktive Üben ohne Einschränkungen durchgeführt werden. Häufig wirkt sich die RAAT sogar positiv auf den muskulären Zustand aus. Manchmal kann eine Spastik die Therapie stören. Die Spastik selbst ist jedoch kein ultimatives Abbruchkriterium. Gerade zu Beginn des Trainings können z.B. die Flexoren des Handgelenks durch die schnelle Bewegung in Dorsalextension des Geräts verstärkt aktiviert werden. Hier kann es sinnvoll sein, vor der Therapie eine Dehnlagerung für die spastische Muskulatur von ca. 30 Minuten durchzuführen (Abb. 7). Der spastische Muskel ist anschließend unempfindlicher gegenüber schnellen Dehnreizen, und das Training kann effektiver starten.

\section{Ausweichbewegungen}

Während des Übens können v.a. bei Geräten mit proximalem Ansatz Ausweichbewegungen über den Rumpf auftreten. Solange die aktive Armbewegung an sich dabei erkennbar ist, müssen diese der Erfahrung nach nicht korrigiert werden. Wird die gesamte Bewegung jedoch nur vom Rumpf ausgeführt, sollte die Bewegung erst einmal leichter gestaltet oder evtl. eine Teilbewegung geübt werden. Beim distalen Ansatz treten Ausweichbewegungen am Rumpf erfahrungsgemäß eher bei Erschöpfung auf und können wie beim proximalen Ansatz zugelassen werden, solange die aktive Bewegung im Unterarm oder Handgelenk erkennbar ist. Ist die Ausweichbewegung zu intensiv, sollte eine Pause eingelegt werden.

\section{Zusammenfassung}

Durch die maschinelle Unterstützung der RAGT und RAAT kann der Patient eine hohe Wiederholungszahl bei aktivem und aufgabenspezifischem Training erreichen. Dadurch werden Arm- und Beinfunktionen sowie auch die Belastbarkeit des Patienten gesteigert. Vor allem akut von Schlaganfall betroffene Patienten, die hinsichtlich der Armfunktionen und der Gehfähigkeit stark eingeschränkt sind, profitieren von der RAGT und RAAT.

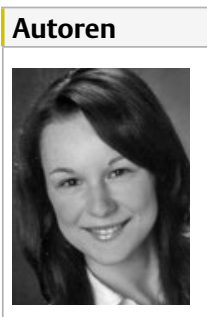

Simone Thomas ist Physiotherapeutin mit Bachelor und Master of Science. Sie promovierte im Jahr 2016. Seit 2012 unterstützt sie das Wissenschaftliche Institut der Klinik Bavaria Kreischa als wissenschaftliche Mitarbeiterin.

Dr. rer. medic. Simone Thomas

Wissenschaftliche Mitarbeiterin

Private Europäische Medizinische Akademie der Klinik Bavaria in Kreischa $\mathrm{GmbH}$

An der Wolfsschlucht 1-2

01731 Kreischa

E-Mail: simone.thomas@klinik-bavaria.de

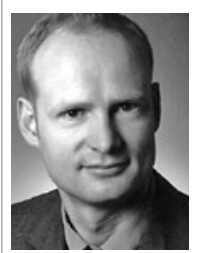

Jan Mehrholz ist Physiotherapeut und Professor für Therapiewissenschaften. Zudem leitet er das Wissenschaftliche Institut der Klinik Bavaria in Kreischa und ist als Privatdozent für Gesundheitswissenschaften/Public Health an der Medizinischen Fakultät der TU Dresden tätig.

Prof. Dr. rer. medic. habil. Jan Mehrholz Klinik Bavaria in Kreischa GmbH

\section{Literatur}

1. Beer S, Aschbacher B, Manoglou D et al. Robot-assisted gait training in multiple sclerosis: A pilot randomized trial. Mult Scler 2008; 14(2): 231-236

2. Dias D, Laíns J, Pereira A et al. Partial body weight support in chronic hemiplegics: A randomized control trial. EuropaMedicophysica 2007; 43(4): 499-504

3. Druzbicki M, Rusek W, Snela S et al. Functional ef fects of robotic-assisted locomotor treadmill therapy in children with cerebral palsy. J Rehabil Med 2013; 45(4): 358-363

4. Gordon C, Wilks R, McCaw-Binns A. Effect of aerobic exercise (walking) training on functional status and health-related quality of life in chronic stroke survivors: A randomized controlled trial. Stroke 2013; 4 1179-1181

5. Hesse S, Tomelleri C, Bardeleben A et al. Robot-assisted practice of gait and stair climbing in nonambulatory stroke patients. J Rehabil Res Dev 2012; 49(4): 613-622

6. Hidler J, Nichols D, Pelliccio M et al. Multicenter randomized clinical trial evaluating the effectiveness of the Lokomat in subacute stroke. Neurorehabil Neural Repair 2009; 23(1): 5-13

7. Karvonen M, Kentala K, Mustala O. The effects of training heart rate: A longitudinal study. Annales Medicinae Experimentalis et Biologiae Fenniae 1957; 3(35): 307-315

8. Mehrholz J, Kugler J, Storch A et al. Treadmill training for patients with Parkinson's disease. Cochrane Database Syst Rev 2015; 8: CD007830

9. Mehrholz J, Pohl M, Platz T et al. Electromechanical and robot-assisted arm training for improving activities of daily living, arm function, and arm muscle strength after stroke. Cochrane Database Syst Rev 2015; 11: CD006876

10. Mehrholz J, Harvey L, Thomas S, Elsner B. Is bodyweight-supported treadmill training or robotic-assisted gait training superior to overground gait training and other forms of physiotherapy in people with spinal cord injury? A systematic review. Spinal Cord 2017. DOI: $10.1038 /$ sc.2017.31

11. Mehrholz J, Thomas S, Werner $C$ et al. Electromechanical-assisted training for walking after stroke (Review). Cochrane Database of Systematic Reviews 2017; Art. No.: CD006185(Issue 5.). DOI: 10.1002/14651858.CD006185.pub4

12. Meyer-Heim A, Borggraefe I, Ammann-Reiffer C et al. Feasibility of roboticassisted locomotor training in children with central gait impairment. Developmental Medicine \& Child Neurology 2007; 49: 900-906

13. Morone G, Bragoni M, losa M et al. Who may benefit from robotic-assisted gait training? A randomized clinical trial in patients with subacute stroke. Neurorehabilitation and Neural Repair 2011; 25(7): 636-644

14. Peurala SH, Airaksinen O, Huuskonen P et al. Effects of intensive therapy using gait trainer or floor walking exercises early after stroke. J Rehabil Med 2009; 41(3): 166-173

15. Pohl M, Werner C, Holzgraefe M et al. Repetitive locomotor training and physiotherapy improve walking and basic activities of daily living after stroke: A single-blind, randomized multicentre trial (DEutsche GAngtrainerStudie, DEGAS). Clin Rehabil 2007; 21(1): 17-27

16. Picelli A, Melotti C, Origano F et al. Robot-assisted gait training in patients with Parkinson disease: A randomized controlled trial. Neurorehabil Neural Repair 2012; 26(4): 353-361

17. Picelli A, Melotti C, Origano F et al. Does robotic gait training improve balance in Parkinson's disease? A randomized controlled trial. Parkinsonism Relat Disord 2012; 18(8): 990-993

18. Smania N, Bonetti P, Gandolfi M et al. Improved gait after repetitive locomotor training in children with cerebral palsy. Am J Phys Med Rehabil 2011; 90(2): 137-149

19. Wu M, Kim J, Gaebler-Spira D et al. Robotic resistance treadmill training improves locomotor function in children with cerebral palsy: A randomized controlled pilot study. Archives of Physical Medicine and Rehabilitation 2017. DOI: 10.1016/j.apmr.2017.04.022

20. Wulf G, Lewthwaite R. Optimizing performance through intrinsic motivation and attention for learning: The OPTIMAL theory of motor learning. Psychon Bull Rev 2016. DOI: 10.3758/s13423-0150999-9

\section{Bibliografie}

DOI 10.1055/s-0043-120323 neuroreha 2017; 9: 180-184

(c) Georg Thieme Verlag KG

Stuttgart · New York · ISSN 1611-6496 